\title{
Propagation-based Content Dissemination for Social Mobile Interactive Multimedia Services
}

\author{
Mu Wang \\ Beijing University of Posts and \\ Telecommunications \\ Beijing, China \\ wangmu@bupt.edu.cn \\ Yiran Wei \\ Beijing University of Posts and \\ Telecommunications \\ Beijing, China \\ wyr100@bupt.edu.cn
}

\author{
Changqiao $\mathrm{Xu}$ \\ Beijing University of Posts and \\ Telecommunications \\ Beijing, China \\ cqxu@bupt.edu.cn \\ Jianfeng Guan \\ Beijing University of Posts and \\ Telecommunications \\ Beijing, China \\ jfguan@bupt.edu.cn
}

\begin{abstract}
With the fast development of mobile communication technologies and increased capabilities of smart terminals, the multimedia service has become one of the most attractive applications in mobile Internet. And the combination of socialbased mobile multimedia and mobile peer-to-peer (P2P) network has attracted increasing research interests in recent years. However, how to deploy large scale P2P-based social multimedia applications over MANETs has become a bid challenge. In this paper, we propose a Propagationbased Content Dissemination solution for Social Interactive Multimedia services over MANETs (PSIM). In PSIM, the video content propagation problem is formulated as an Epidemic Information Dissemination (SIR) model. Based on the chunk-based dissemination model and analysis, a novel Propagation-based Video Chunk Demands Estimation Algorithm and a Stability-based Cooperative Carrier Selection Mechanism are proposed to estimate the video chunk demands and choose stable cooperative cache nodes, respectively. Simulation results show how PSIM achieves better performance in comparison with another state-of-art solution.
\end{abstract}

\section{Keywords}

Social Network, Interactive Multimedia, Cooperative Cache, MANETs

\section{INTRODUCTION}

Mobile Ad-hoc Networks(MANETs) has become a significant wireless communication technology in future heterogeneous network environment due to its robust, scalability and self-organizing[1][2][3][4]. Recent years, the Internet has wit- nessed the blossom of social network applications (eg. Renren, Facebook), and a large number of new social-based applications have been fostered into MANETs, including social interactive multimedia service[5][6][7]. Unlike the traditional multimedia streaming service, the interactive multimedia services allow user's VCR-like operations and provide interactions between users with social relationship, which meets the demands of user free access to the interested video content. According to Wang et al. [8], social interactive multimedia services will dominate the future multimedia traffic over Internet. P2P technologies make use of users' upload bandwidth to compensate the bandwidth of tradition $\mathrm{C} / \mathrm{S}$ architecture, which has become a main trend for future VoD service[9][10]. How to provide high quality P2P-based social interactive multimedia in wireless environment has become a hot topic[11, 12, 13]. Wang et al in [14] have showed that the user preference of video has small-world characteristics. Shen et.al in [9] proposed Social-Tube, including localityaware video pre-fetching mechanism, the chunk-based delivery abilities enhance policies, and buffer management algorithm. In [15], Cheng et.al designed a social media sharing system which utilizes social reciprocity to increase the user sharing efficiency, and employs peer-assisted designs to distribute video streams with low server cost.

Due to the high dynamic of wireless environment, how to deploy large scale P2P-based social applications over MANETs has become a big challenge, numerous solutions have proposed. In [16], Xu et.al proposed a novel P2P-based video sharing solution over VANETs by using $4 \mathrm{G}$ networks as a compensate. In [17], Chen et.al proposed SPOON, a community-based P2P content file sharing solution in disconnected mobile ad-hoc networks, groups the users which have common interests in shared files and frequent interactions as a community. Bandara et.al in [18] proposed a community-based cache mechanism to enhance both communitywide and system-wide lookup performance. In [19], a novel ant-inspired mini-community-based video sharing solution (AMCV) has been proposed for on demand services in wireless mobile networks. In AMCV, a multiple minicommunities based on the video content is constructed which aims to reduce resource maintenance costs and enhanced the 


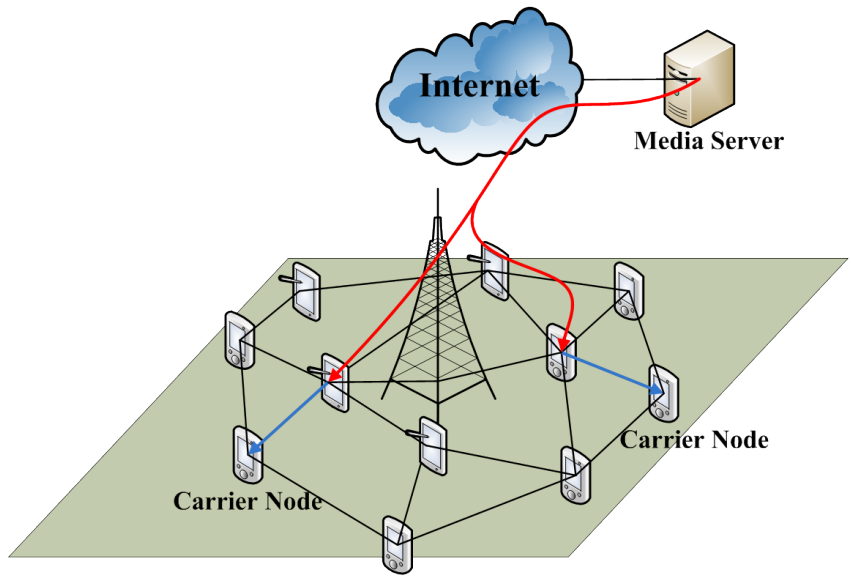

Figure 1: The architecture of PSIM

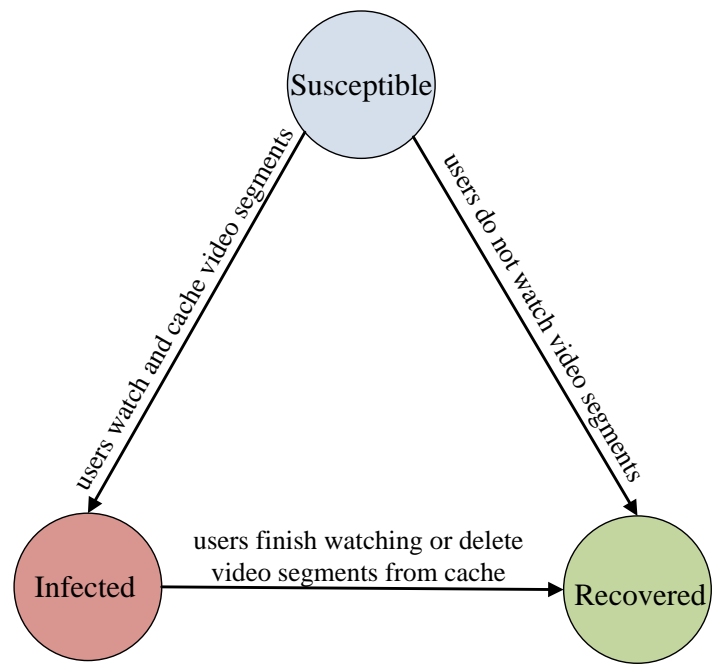

Figure 2: Propagation mechanism based on SIR model

types: Susceptible, noted by $S$, represents the nodes which have some probabilities to be infected. Infected, noted by $I$, represents the nodes which have been infected and tend to infect others. And Recoverd, noted by $R$, represents the nodes that have been cured or already died, which means the recovered nodes cannot affect on the spread of diseases. Fig. 2 shows the video propagation mechanism based on the SIR model. The propagation mechanism is defined as in Eq. (1):

$$
\left\{\begin{array}{l}
S(i)+I(j) \stackrel{\lambda}{\rightarrow} I(i)+I(j) \\
I(i) \stackrel{\mu}{\rightarrow} R(i)
\end{array}\right.
$$

In interactive multimedia system, the node which gets interested in video segment $c_{i}$ can be defined as infected node $S^{c_{i}}(h)$, the node which plays or caches the video segment $c_{i}$ can be defined as $I^{c_{i}}(h)$, and the node which has already finished playing the video segment $c_{i}$ can be defined as $R^{c_{i}}(i)$. Suppose at time $t$, the density for these three kinds of nodes are $s^{c_{i}}(t), i^{c_{i}}(t), r^{c_{i}}(t)$, we can get differential equations as in Eq. (2) :

$$
\left\{\begin{array}{l}
\frac{d s^{c_{i}}(t)}{d t}=-\lambda s^{c_{i}}(t) i^{c_{i}}(t) \\
\frac{d i^{c_{i}}(t)}{d t}=\lambda s^{c_{i}}(t) i^{c_{i}}(t)-\mu i^{c_{i}}(t) \\
\frac{d r^{c_{i}}(t)}{d t}=\mu i^{c_{i}}(t)
\end{array}\right.
$$

where $\lambda$ represents the access rate of the video segment, $\mu$ represents the recovered rate, and the constraint condition is $s^{c_{i}}+i^{c_{i}}+r^{c_{i}}=1$. Through this model, a specific trend can be concluded about the scale of infected users, which play or cache video content during the video propagation process in interactive multimedia system. At first, infected users increase generally with more and more users get interested in the video content. However, if infected users finish playing video, they will transfer to recovered users. On the other hand, the susceptible users, who get interested in video content, will decrease as time goes by. Therefore, the infected users in system will tend to a constant value finally. Extend 
Eq. (2) into Eq. (3) with initial conditions $s^{c_{i}}(0), i^{c_{i}}(0)$ to describe the propagation rule in interactive multimedia system:

$$
\left\{\begin{array}{l}
\frac{d s^{c_{i}}(t)}{d t}=-\lambda s^{c_{i}}(t) i^{c_{i}}(t) \\
\frac{d i^{c_{i}}(t)}{d t}=\lambda s^{c_{i}}(t) i^{c_{i}}(t)-\mu i^{c_{i}}(t) \\
\frac{d r^{c_{i}}(t)}{d t}=\mu i^{c_{i}}(t) \\
s^{c_{i}}(0)=s_{t_{0}}^{c_{i}}, i^{c_{i}}(0)=i_{t_{0}}^{c_{i}},
\end{array}\right.
$$

where $t_{0}$ denotes the initial time, $s_{t_{0}}^{c_{i}}$ denotes the density for users get interested to video segment $c_{i}$, and $i_{t_{0}}^{c_{i}}$ denotes the density for users who play or cache video segment $c_{i}$. Since solving analytical solutions for $i^{c_{i}}(t)$ and $s^{c_{i}}(t)$ is very complicated, the usual method is to find numerical solutions [21] for Eq. (3). At time $t$, the relationship between density for users playing video segment $c_{i}$ and users get interested to video segment $c_{i}$ can be defined in Eq. (4):

$$
i^{c_{i}}(t)=s_{t_{0}}^{c_{i}}+i_{t_{0}}^{c_{i}}-s^{c_{i}}(t)+\frac{\mu}{\lambda} \operatorname{In} \frac{s^{c_{i}}(t)}{s_{t_{0}}^{c_{i}}}
$$

According to eq. (4), we can obtain the $s^{c_{i}}(t)$ as follow:

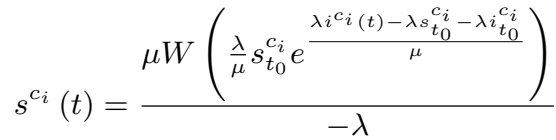

where W represents Lambert W Function [12]. Therefore, the density for users get interested to video segment $c_{i}$ can be calculated by Eq. (5) based on the SIR model and the knowledge of initial situation for interactive multimedia system. Moreover, the number of cached video segments can be determined to achieve requirements for users.

\section{PSIM DETAILS DESIGN}

In this section, we present two practical algorithms to solve the above video dissemination problem in realistic environment: Propagations-based Video Chunk Demands Estimation Algorithm and Stability-based Cooperative Carrier Selection Mechanism. Due to the difficulties of obtaining parameters of Eq. (5) and gathering information in a mobile distribute environment, Propagations-based Video Chunk Demands Estimation Algorithm has been proposed to analyse the video demands in real time over the MANETs. And Stability-based Cooperative Carrier Selection Mechanism aim$\mathrm{s}$ to determine the nodes to carry the corresponding video content, which ensures the network resource can be utilized more efficiently.

\subsection{Propagations-based Video Chunk Demand- s Estimation Algorithm}

Based on the video dissemination analysis above, we present a practical algorithm: Propagations-based Video Chunk Demands Estimation Algorithm. In PSIM, the media server not only provides the initial video content for mobile user, but also manages the users' query information of video chunks by using a list. Unlike the traditional peer-assisted video sharing system, each mobile node in PSIM maintains a query information table to record the request information they received, the query information of node $U_{i}$ can be describe as

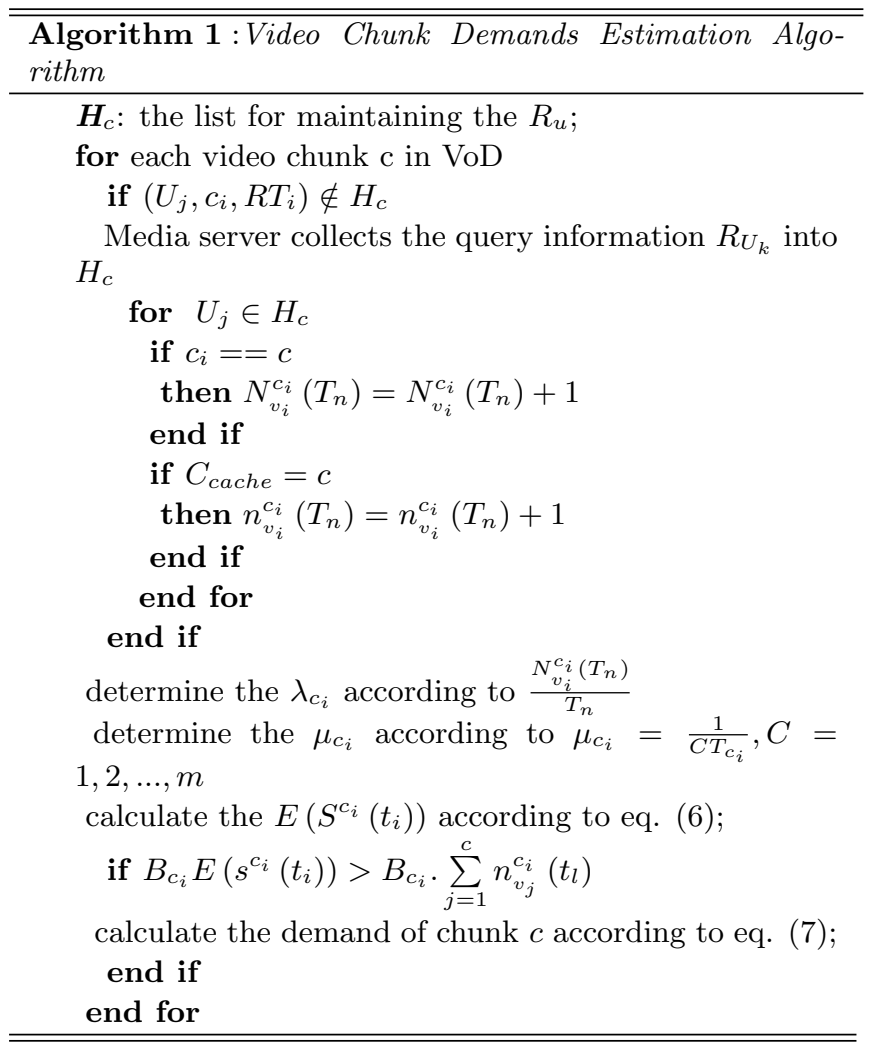

following 4-tuple: $R_{U_{k}}=\left(U_{j}, c_{i}, R T_{i}, C_{\text {cache }}\right)$, where $U_{j}$ represents the request user ID, $c_{i}$ represents the request video chunk, $R T_{i}$ represents the request time, and $C_{\text {cache }}$ represents the current video chunk that $U_{i}$ cache. For every time interval $T$, the mobile nodes upload the received query information to media server, where $T=n T_{c}, n=1,2, \ldots, n$, and $T_{c}$ is the playback time of video chunk. In order to predict the future demands of video chunk $k$, we use $n_{v_{i}}^{c_{i}}\left(T_{n}\right)$ denotes the number of users which cache video chunk $c_{i}$ in current period $T_{n}$, and $n_{v_{i}}^{c_{i}}\left(T_{n}\right)$ can be calculate by the $R_{U_{k}}$. In practical environment, the $\lambda, \mu$ in eq. (5) are hard to obtain the accurate values, in order to implement the algorithm in real world, the media server use $\frac{N_{v_{i}}^{c_{i}}\left(T_{n}\right)}{T_{n}}$ to approximate the access rate $\lambda$ in this paper, where $N_{R}^{c_{i}}\left(T_{n}\right)$ represents the number of requests of video chunk $c_{i}$ in time interval $T_{n} . N_{R}^{c_{i}}\left(T_{n}\right)$ can be calculate according to query information that media server gathered. As the same reason, the recovered rate is set to: $\mu_{c_{i}}=\frac{1}{C T_{c_{i}}}, C=1,2, \ldots, m$, where $C$ denotes the size of the cache of the node, which should be the integral multiple of the size of video segment. Based on the defined diffusion rate, recovered rate and Eq. (5), the number of users who get interested in video segment $c_{i}$ at time $T_{n}+1$ can be defined as

$$
E\left(S^{c_{i}}\left(T_{n+1}\right)\right)=\frac{N_{\text {vod }} \mu_{c_{i}} W\left(\frac{\lambda_{c_{i}}}{\mu_{c_{i}}} E\left(s_{t_{0}}^{c_{i}}\right) e^{f\left(T_{n}\right)}\right)}{-\lambda_{c_{i}}}
$$

where $f\left(T_{n}\right)=\frac{1}{\mu_{c_{i}}}\left\{\lambda_{c_{i}}\left(n_{v_{i}}^{c_{i}}\left(T_{n}\right)+N_{v_{i}}^{c_{i}}\left(T_{n}\right)-n_{v_{i}}^{c_{i}}\left(T_{n}\right) \mu_{c_{i}}\right)\right.$ $\left.-\lambda_{c_{i}} E\left(s^{c_{i}}\left(t_{0}\right)\right)-\lambda_{c_{i}} N_{v_{i}}^{c_{i}}\left(T_{0}\right)\right\}, N_{\text {vod }}$ represents the total number of users in the VoD system. Suppose the bit rate is $B_{c_{i}}$, the bandwidth for the demand for video segment $c_{i}$ at time 
$t_{i}$ is $B_{c_{i}} E\left(s^{c_{i}}\left(t_{i}\right)\right)$. The current system can provide bandwidth $B_{c_{i}} . N_{v_{i}}^{c_{i}}\left(T_{n}\right)$. If $B_{c_{i}} E\left(s^{c_{i}}\left(t_{i}\right)\right)>B_{c_{i}} . N_{v_{i}}^{c_{i}}\left(T_{n}\right)$, more cache backups are needed. The demand video chunk number $n_{r}^{c_{i}}$ can be expressed as

$$
n_{r}^{c_{i}}=\frac{B_{c_{i}} E\left(s^{c_{i}}\left(t_{i}\right)\right)-B_{c_{i}} \cdot \sum_{j=1}^{c} n_{v_{j}}^{c_{i}}\left(t_{l}\right)}{B_{\text {upload }}}
$$

where $B_{\text {upload }}$ denotes the upload bandwidth. Therefore, media server should choose $n_{r}^{c_{i}}$ nodes which cache the video chunk $c_{i}$ according to Eq. (7). The details algorithm is described as Algorithm 1.

As the algorithm discussed, the media server maintains a list $H_{c}$ to gather the query information $R_{U}$, and the end user uploads the $R_{U}$ periodically. The media server leverages the upload $R_{U}$ to calculate the $\lambda_{c_{i}}$ and $\mu_{c_{i}}$ according to $\lambda_{c_{i}}=\frac{N_{v_{i}}^{c_{i}\left(T_{n}\right)}}{T_{n}}$ and $\mu_{c_{i}}=\frac{1}{C T_{c_{i}}}$. After the approximate value of $\lambda_{c_{i}}$ and $\mu_{c_{i}}$ obtained, the media server estimates the demand of each video chunk according to Eq. (7). Therefore, we can obtain the video demands in a large scale distribution environment through this algorithm.

\subsection{Stability-based Cooperative Carrier Selec- tion Mechanism}

In PSIM, a number of mobile nodes called carriers are selected. For carrier nodes, the buffer can be divided into two part: playback buffer and cooperative buffer. The playback buffer caches the current playback video chunks of users, which is the same as ordinary nodes. While the cooperative buffer aims to cache the popularity video chunks according to Eq. (7). In order to make full use of users' free storages and to guarantee the smooth playback for users, the system should choose the stable nodes to act as carriers, we first define the stability of nodes $\mathrm{j}$ as follows:

$$
s t_{j}=\frac{t_{j}}{p_{v}}
$$

where $t_{j}$ is the joint time of nodes and $t$ is the playback time of video. The media server records the stability of nodes as $S T=\left(s t_{1}, s t_{2}, \ldots, s t_{n}\right)$ in descend order. For each video chunk $c_{i}$, the number of required carriers is $n_{r}^{c_{i}}$. For the chunk $c_{i}$ which has the largest $n_{r}^{c_{i}}$ value chooses the first $n_{r}^{c_{i}}$ of $S T$ nodes as carriers. The rest video chunks can be done follow the same method. This is because the more popular chunks should be cached by more stable carriers, which can guarantee the request success rate of popular video chunks. The detailed algorithm is described as Algorithm 2.

As the algorithm described, the aim of Stability-based Cooperative Carrier Selection Mechanism is to choose the relatively stable mobile nodes in the system to cache video contents with high demand. The media server maintains lists JT and $C_{p}$. JT is to record the join time of mobile node and $C_{p}$ is to record the demand of video content. The media server sorts the video content demands in descend order and calculates the number of carrier nodes required for each chunk, choose the highest stability nodes in ST as the carrier of corresponding video chunk, and send messages to

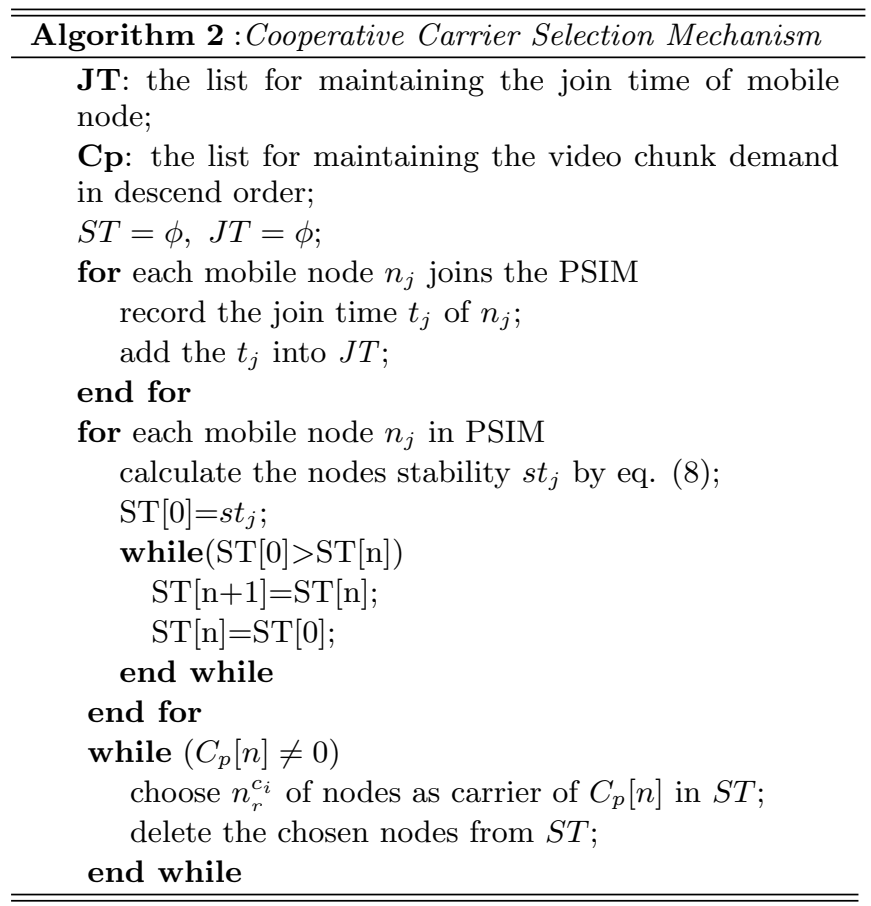

these mobile nodes to cache the video content.

\section{PERFORMANCE EVALUATION}

We compare the performance of the proposed solution PSIM with the popularity-based balanced chunk distribution algorithm of PMCV [7]. Both systems are deployed in a VANET-based environment by making use of the Network Simulator (NS2). We choose a 10 minutes long video clip and evenly divided it into 20 chunks, namely the length of each chunk is $30 \mathrm{~s}$, the detailed simulation settings and performance comparison results are described as follows.

\subsection{Testing Topology and Scenarios}

Table 1 lists some important NS2 simulation parameters of the VANET. There are 1000 mobile nodes in VANET. The nodes are deployed in a square area of $\mathrm{x}=2000 \mathrm{~m}$ by $\mathrm{y}=2000 \mathrm{~m}$. An urban topology, which consists of five horizontal and five vertical streets is considered. Every street has two lanes in each direction. The vehicular movement behavior follows the Manhattan mobility model[22]. In Manhattan mobility model, vehicles are moving along the street. When a vehicle reaches the specified destination, it is assigned a random residence time and restarts its movement with a new assigned speed and destination. Both solutions use the IEEE 802.11p WAVE network interface which only supports wireless communications between vehicle nodes for the exchange of control and state information and the transmission of video data, and the bandwidth of end user is set to $27 \mathrm{Mbps}$. We also create 20,000 synthetic user viewing $\log$ entries based on the interactive actions, measurements and statistics from[23]. The mobile nodes which finish the playback of the whole video will quit the system. The bandwidth at the media server side is set to $10 \mathrm{Mb} / \mathrm{s}$. The $n$ of $T=n T_{c}$ is set to 3 according to the repeated test results. 
Table 1: Simulator Parameter Setting

\begin{tabular}{|l|l|}
\hline Parameters & Value \\
\hline Area & $2000 \times 2000 m^{2}$ \\
Channel & Channel/Wireless Channel \\
Network Interface & Phy /WirelessPhyExt \\
MAC Interface & Mac/802.11 \\
bandwidth & $27 \mathrm{Mbps}$ \\
Frequency & $5.9 \mathrm{GHz}$ \\
Multiple Access & OFDM \\
Transmission Power & $33 \mathrm{dBm}$ \\
Wireless Transmission Range & $250 \mathrm{~m}$ \\
Interface Queue Type & Queue/DropTail/PriQueue \\
Interface Queue Length & 50 packets \\
Antenna Type & Antenna/OmniAntenna \\
Routing Protocol & DSR \\
Min mobile speed & Om/s \\
Max mobile speed & $30 \mathrm{~m} / \mathrm{s}$ \\
Network layer protocol & IP protocol \\
Transport layer protocol & TCP \\
\hline
\end{tabular}

The cache size of mobile node is set to 4 .

\subsection{Performance Evaluation}

The performance of PSIM is compared with that of PM$\mathrm{CV}$ in terms of average chunk seek delay (ACSD), average lookup success rate (ALSR), server stress and control overhead, respectively. Besides, in order to estimate the quality of experience (QoE) metric of PSIM, we compare the peak signal-to-noise ratio (PSNR) of PSIM with PMCV.

Average Chunk Seek Delay (ACSD): The mean difference between the time when the node receives the video data and the time when the node sends the request message indicate the average chunk seek delay. Fig. 3 plots the comparison results of PSIM and PMCV with the increased number of mobile nodes. $\mathrm{PMCV}^{\prime} \mathrm{S}$ ACSD curve reaches the lowest value of $0.792 \mathrm{~s}$, after which increases to roughly $1.2 \mathrm{~s}$. This is because the increased mobile nodes reduce the cross community video chunk requests, therefore, the $\mathrm{PMCV}^{\prime} \mathrm{s}$ ACSD decrease before 140. However, with the system scale keeps growing, the network congestion level goes up, the whole system suffers a long delivery latency and high packet loss rate, thus the ACSD increases rapidly. The PSIM follows the same trends, but unlike the popularity-based balanced chunk distribution algorithm (PMCV), PSIM analyses the evolution pattern of user playback behavior, which estimates the demand of each video chunk, and proposes a carrier selection mechanism which chooses the stable mobile nodes to cache video chunk with big demand. Therefore, the PSIM outperforms PMCV.

Average lookup success rete (ALSR): A node receives the data of requested chunk from other nodes after it sends first request message. The ratio between the number of successful lookup times and the number of total lookup times is considered as ALSR. Fig. 4 plots the ALSR comparison results of PSIM and PMCV with the increased number of mobile nodes. As figure shows, with the mobile nodes number increases, both PSIM and PMCV increase the ALSR rapidly. Since PSIM analyses the video chunk demand

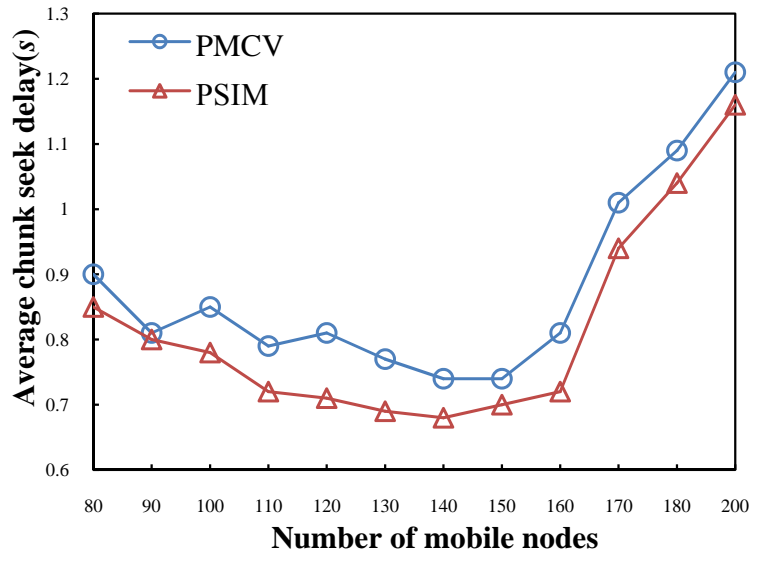

Figure 3: Average seek delay versus mobile nodes number

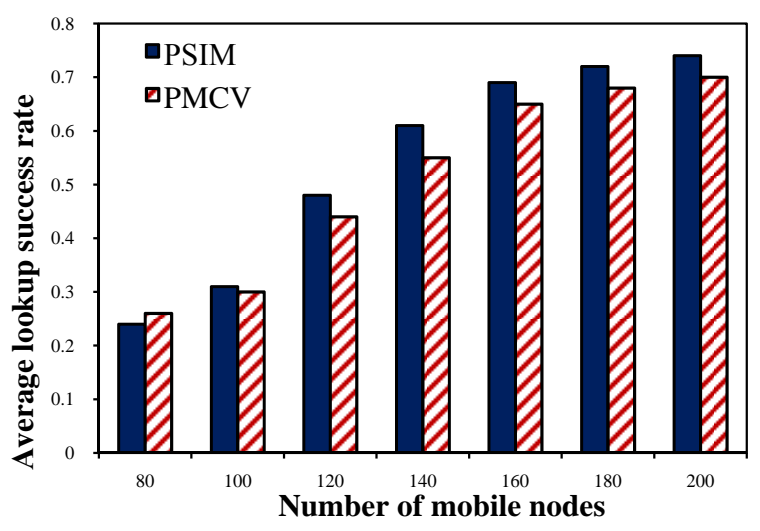

Figure 4: ALSR versus mobile nodes number

by building a SIR based model, and proposes a mechanis$m$ which ensures the popular video contents will be cached in relatively stable nodes, the resource distribution is more efficient and can better suit the dynamic network situation. Therefore, the PSIM can obtain the high level ALSR.

Server Stress: The ratio between the current number of streams serving mobile nodes at the media server side and the maximum number of streams supported by the server is defined as the server stress. Fig. 5 plots the server stress comparison results of PSIM and PMCV with the increased number of mobile nodes. Due to the system scale is small at the beginning, most of the users' requests aare responded by the media server. Then with the increase of mobile nodes number, the probability of video chunk cache in mobile nodes also increases, therefore the server stress decreases rapidly. $\mathrm{PMCV}^{\prime}$ s popularity-based balanced chunk distribution algorithm only focuses on current video demand, ignores user request behavior with time varying. PSIM efficiently estimates the video chunk demand and considers the carrier stability, which ensures accurate pre-fetching of video chunks in mobile environment, thus the PSIM obtains the lower server stress than PMCV.

Control overhead: The bandwidth which is used for control message exchange per second is defined as control over- 

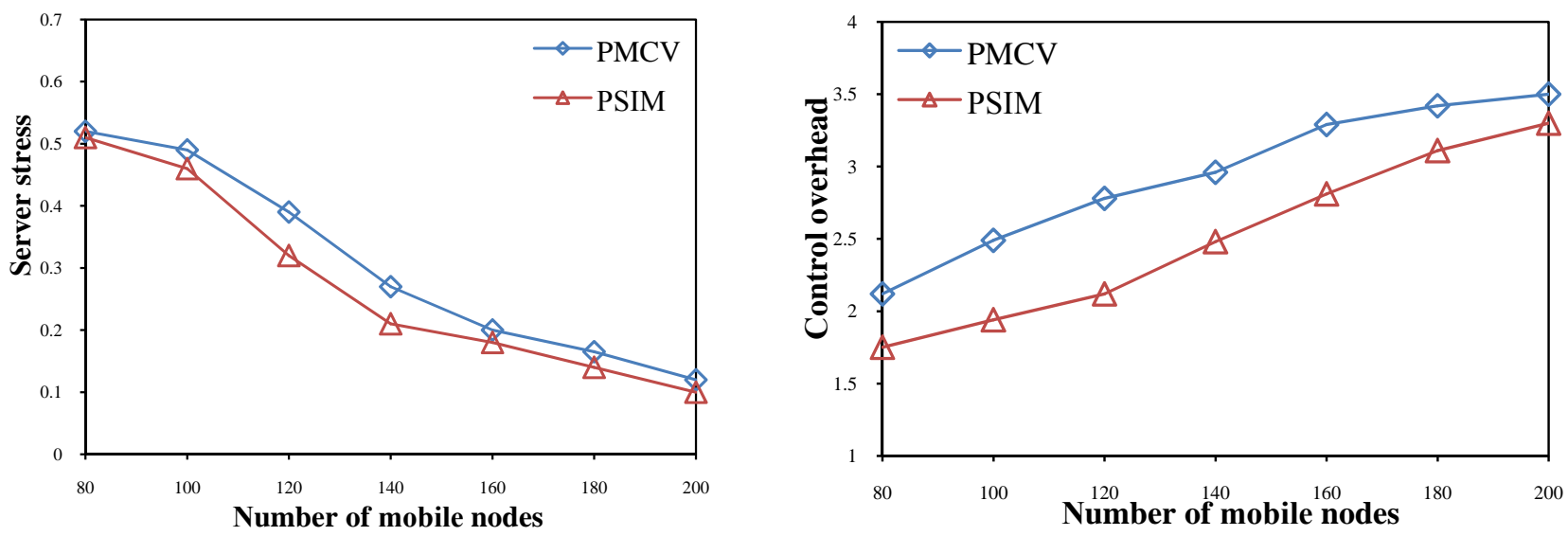

Figure 5: Server stress versus mobile nodes number Figure 6: Control overhead versus mobile nodes number

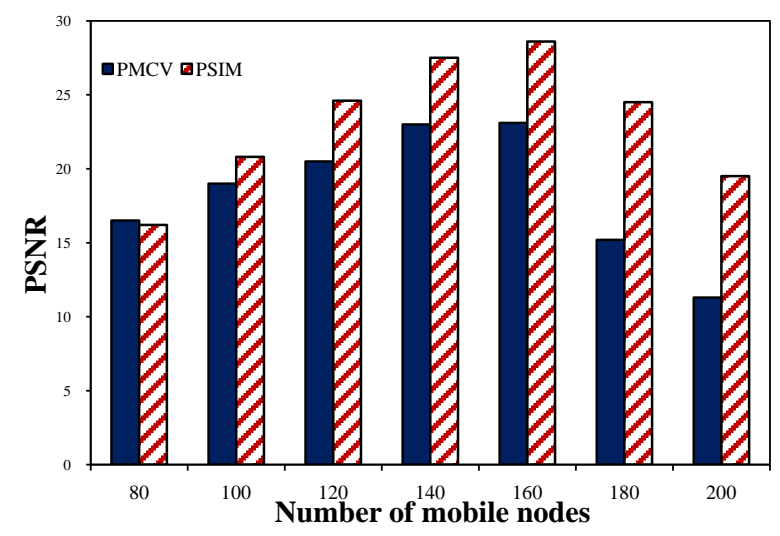

Figure 7: PSNR versus mobile nodes number

head. Fig. 6 shows the control overhead comparison results with the increase in the number of vehicles from 80 to 200 . Due to the system scale increase, the curve corresponding to both solutions maintains a fast increasing trend with increasing number of system members. The curve of PMCV shows a faster rise trend than PSIM. When the number of mobile nodes reaches 120, the deviation between two solutions reaches the peak value as $0.66 \mathrm{~kb} / \mathrm{s}$. The content caching mechanism in PMCV depends on user playback pattern, with the video play, the playback of user is varying, thus to ensure the accurate, the nodes should exchange the playback pattern frequently. In PSIM, mobile nodes upload the query information to media server every time interval $T$. Consequently, the control overhead of PSIM is lower than PMCV.

Peak Signal-to-Noise Ratio (PSNR): In this paper, in order to estimate the received video quality of end user, we use the peak signal-to-noise ratio (PSNR) as the video quality metric. The PSNR estimation rule is according to [7]. In this paper, we estimate the average PSNR of each mobile nodes in system. Fig. 7 shows the PSNR comparison results with the increase of the number of vehicles from 80 to 200 . As it shows in the figure, both PMCV and PSIM experience an increasing trend before the number of mobile nodes reaches 160 . However, with the system scale keeps expand- ing, the PSNR of PMCV declines sharply. This is due to the congestion caused by the increase number of mobile nodes in mobile network, and which has obvious impact on QoE of end users. The PSIM declines from 160 to 200 with the same reason, but the curve of PSIM declines more smoothly. This is because PSIM employs a novel video chunk demands estimation algorithm based on video dissemination model to cache popular video content advanced. Moreover, PSIM utilizes the network resource more efficiently. Consequently, the PSNR of PSIM is better than PMCV.

\section{CONCLUSIONS}

This paper proposes a novel Propagation-based Content Dissemination solution for Social Interactive Multimedia over MANETs (PSIM). PSIM formulates the video chunk dissemination as a SIR model. According to the SIR model, we obtain the interactive multimedia system's video demands evolution with time varying. Based on the problem formulation, we propose a Propagationsbased Video Chunk Demands Estimation Algorithm to estimate the video demands in realistic environment, and introduce a Stability-based Cooperative Carrier Selection Mechanism to choose proper nodes to cache the video chunks. Simulation results show how PSIM outperform PMCV in terms of the average chunk seek delay, average lookup suc- 
cess rate, server stress, control overhead, and peak signalto-noise ratio, respectively.

\section{ACKNOWLEDGMENTS}

This work was supported in part by the National Natural Science Foundation of China under Grants 61372112 and 61232017, in part by the Beijing Natural Science Foundation under Grant 4142037, in part by the National Key Basic Research Program (973 Program) of China under Grant 2013 CB329102.

\section{REFERENCES}

[1] L. Zhou, B. Zheng, A. Wei, B. Geller, and J. Cui. A robust resolution-enhancement scheme for video transmission over mobile ad-hoc netwroks. IEEE Transactions on Brodacasting, 54(2):312-321, June 2008.

[2] C. Xu, Z. Li, J. Li, H. Zhang, and G.-M. Muntean. Cross-layer fairness-driven concurrent multipath video delivery over heterogenous wireless networks. IEEE Transactions on Circuits and Systems for Video Technology, PP(99), December 2014.

[3] Z. Wang, Y. Chen, and C. Li. Psr: A lightweight proactive source routing protocol for mobile ad hoc networks. IEEE Transactions on Vehicular Technology, 63(2):859-868, February 2014.

[4] C. Xu, T. Liu, J. Guan, H. Zhang, and G.-M. Muntean. Cmt-qa: Quality-aware adaptive concurrent multipath data transfer in heterogeneous wireless networks. IEEE Transactions on Mobile Computing, 12(11):2193-2205, November 2013.

[5] X. Cheng, H. Li, and J. Liu. Video sharing propagation in social networks: Measurements, modeling and analysis. in Proc. IEEE INFOCOM, pages 45-49, 2013.

[6] Z. Wang, L. Sun, W. Zhu, and S. Y. Joint social and content recommendation for user-generated videos in online social networks. IEEE Transactions on Multimedia, 15(3):698-709, April 2013.

[7] C. Xu, S. Jia, M. Wang, L. Zhong, H. Zhang, and G.-M. Muntean. Performance-aware mobile community-based vod streaming over vehicular ad hoc networks. IEEE Transactions on Vehicular Technology, 64(3):1201-1217, March 2015.

[8] X. Wang, M. Chen, T.T. Kwon, L.Y. Yang, and V. M. Leung. Ames-cloud: A framework of adaptive mobile video streaming and efficient social video sharing in the clouds. IEEE Transactions on Multimedia, 15(4):811-820, June 2013.

[9] H. Shen, Z. Li, Y. Lin, and J. Li. Socialtube: $\mathrm{p} 2 \mathrm{p}$-assisted video sharing in online social networks. IEEE Transactions on Parallel and Distributed Systems, 25(9):2428-2440, September 2014.

[10] C. Chang, W. Chen, and C. Hung. Reliable consideration of $\mathrm{p} 2 \mathrm{p}$-based vod system with interleaved video frame distribution. IEEE Systems Journal, 8(1):304-312, March 2014.

[11] H. Li, H. Wang, J. Liu, and K. Xu. Video requests from online social networks: Characterization, analysis and generation. in Proc. IEEE INFOCOM, pages 50-54, 2013.
[12] D. Ho and H. Song. Resource allocation algorithm based on social relation for video streaming services over p2p network. in IEEE International Conference on Networks (ICON), pages 185-190, 2012.

[13] A. O. Nwana, S. Avestimehr, and T. Chen. A latent social approach to youtube popularity predictionn. in IEEE GLOBECOM, pages 3138-3144, 2013.

[14] Z. Wang, C. Wu, L. Sun, and S. Yang. Peer-assisted social media streaming with social reciprocity. IEEE Transactions on Network and Service Management, 10(1):84-94, March 2013.

[15] X. Cheng, JC. Liu, and C. Dale. Understanding the characteristics of internet short video sharing: A youtube-based measurement study. IEEE Transactions on Multimedia, 15(5):1184-1194, Auguest 2013.

[16] C. Xu, F. Zhao, J. Guan, H. Zhang, and G.-M. Muntean. Qoe-driven user-centric vod services in urban multi-homed p2p-based vehicular networks. IEEE Transactions on Vehicular Technology, 62(5):2273-2289, June 2013.

[17] K. Chen, H. Shen, and H. Zhang. Leveraging social networks for $\mathrm{p} 2 \mathrm{p}$ content-based file sharing in disconnected manets. IEEE Trans. Mobile Comput, 13(2):235-249, February 2014.

[18] H. M. N. D. Bandara and A. P. Jayasumana. Community-based caching for enhanced lookup performance in $\mathrm{p} 2 \mathrm{p}$ systems. IEEE Transactions on Parallel and Distributed systems, 24(9):1698-1710, September 2013.

[19] C. Xu, S. Jia, L. Zhong, H. Zhang, and G.-M. Muntean. Ant-inspired mini-community-based solution for video-on-demand services in wireless mobile networks. IEEE Transactions on Broadcasting, 60(2):322-335, June 2014.

[20] P. Chen, S. Cheng, and K. Chen. Optimal control of epidemic information dissemination over networks. IEEE Transactions on Broadcasting, 44(12):2316-2328, December 2014.

[21] E. Lsaacson. Analysis of numerical methods. In Courier Dover Publications.

[22] F. Bai, N. Sadagopan, and A. Helmy. The important framework for analyzing the impact of mobility on performance of routing protocols for ad hoc networks. Ad Hoc Networks, 1(4):383-403, November 2003.

[23] A. Brampton, A. MacQuire, I. A. Rai, N. J. P. Race, L. Mathy, and M. Fry. Characterising user interactivity for sports video-on-demand. in Proc. ACM NOSSDAV, Urbana-Champaign, IL, pages 1-6, April 2007. 